\title{
Estimating Physical Properties of Confirmed Exoplanets: I. Calculation of essential planetary properties-Possible M-R cataloging of exoplanets
}

\author{
Bijan Nikouravan \\ Department of Physics, Islamic Azad University (IAU), Varamin - Pishva Branch, Iran
}

Received March 2021

Received in revised: April 2021

Published: April 2021

\begin{abstract}
The discovery of extrasolar planets outside our solar system and around other stars is now well underway. In the presented paper, calculations of some physical properties for confirmed exoplanets have been done. We have estimated physical properties such as the semi-major axis for potentially habitable exoplanets, the mass of planets by applying Kepler's third law around the mass of solar, Jupiter, and Earth-mass, stellar luminosity, habitability zone for the inner center and outer regions, radial velocity amplitude, planetary equilibrium temperature (PET) or effective radiation emission temperature and planet density. The massradius (MR) relationship of planets was investigated for potentially habitable exoplanets of three different groups of extrasolar planets: Subterran (Mars-size), Terran (Earth-size), and Superterran (Super-Earths or Mini-Neptunes); introduced in PHL, and found well coefficient values for each group. The minimum and maximum values for the mass and radius of exoplanets have been selected from $0.1<M<10 M_{\oplus}$ and $0.4<R<2.5 R_{\oplus}$. The same MR relationship has also estimated the same properties for a larger number of confirmed exoplanets with a mass and radius of $0.1<\mathrm{M}<100 M_{\oplus}$ and $0.4<R<15 R_{\oplus}$, respectively, resulting their classification within 7 groups of mass and radius, with good coefficient values for each group. This is a new, possible cataloging that may need more effort for concluding a better understanding of the properties and varieties of the exoplanets.
\end{abstract}

Keywords: Planetary system - extrasolar planets - physical properties of exoplanets - Mass-Radius relationship - exoplanet classification

C2021 The Authors. Published by Fundamental Journals. This is an open-access article under the CC BY-NC https://creativecommons.org/licenses/by-nc/4.0/

\section{INTRODUCTION}

Fundamental quantities such as mass, radius, effective temperature, semimajor axis, luminosity, etc., are essential to study planets, stars, and widely for particular Extrasolar systems and Exoplanets. Traditionally with the study of relations between these types of fundamental quantities, scientists can acquire more knowledge and information not only about our solar system but also for other celestial objects outside our solar system such as extrasolar systems and 
exoplanets. Depending on the improvements in theoretical and observational data, many relations have been developed and modified many times from 20 years ago till today.

Relatively recent studies on the subject are by (Bashi, Helled, Zucker, Mordasini, \& Astrophysics, 2017; Malhotra, 2015; Seager, Kuchner, Hier-Majumder, \& Militzer, 2007; Weiss \& Marcy, 2014). To date, quite several advances have been made on both sides; theoretical and observational. On the theoretical side, extrasolar planets (Baraffe, Chabrier, \& Barman, 2009; Perryman, 2000), and in theoretical vs observational uncertainties (Müller, Ben-Yami, \& Helled, 2020), and experimental, physical properties of extrasolar planets physics has undergone appreciable revision. So now the problem with this huge amount of data and information is that we need to find out more related to these fundamental physical quantities to answer our unknown questions not only about the solar system but also for outside of our solar system as extrasolar planets and extrasolar systems.

To do this, we first consider the calculation of some physical properties of exoplanets such as the Semi-major axis for 35 potentially habitable exoplanets, the mass of planets by applying Kepler's third law, around the mass of solar, Jupiter, and Earth-mass, stellar luminosity, and radial velocity amplitude. The MR relationship is the next aim of this paper for potentially habitable exoplanets of the suggested three groups in the Planetary Habitability Laboratory (PHL) (Planetary Habitability Laboratory, 2021). The minimum and maximum values for the mass and radius of exoplanets have been selected from $0.1<M<10 M_{\oplus}$ and $0.4<R<$ $2.5 R_{\oplus}$

\section{DATA}

All data used in this paper is from two data centers. The first one is "The NASA Exoplanet Archive" (NASA Exoplanet Archive, 2021) which is an online directory and astronomical planet and data service that collects and provides public data that supports the search and description of exoplanets and their host stars (Akeson et al., 2013). The second is "The Habitable Exoplanets Catalog" which is also the next online source of data and catalog as PHL (Planetary Habitability Laboratory, 2021) for MR relationship. The PHL is managed by the University of Puerto Rico at Arecibo. All data collected are updated by end of April 2021.

\section{SEMI-MAJOR AXIS CALCULATION}

One of the most important and essential physical properties is the semi-major axis of planets. Here, the calculation of the semi-major axis of 35 confirmed and habitable exoplanets have been done. All data were necessary for this calculation, collected from (NASA Exoplanet Archive, 2021).

Newton's modification of Kepler's third law applied for the host stars and orbital period of planets. Here $M_{S}$ is the mass of a star, $M_{p}$ is the mass of exoplanets, $P$ is the orbital period of a planet moving around the center of mass of its star, and finally, $a$ is the semi-major axis of the planet in the AU unit as shown in Equation (1).

$$
\left(M_{s}+M_{p}\right) P^{2}=a^{3}
$$

Typically, the mass of planets is very small in comparison with the mass of stars $\left(M_{p} \ll M_{s}\right)$ therefore equation (1) reduces to,

$$
M_{S} P^{2}=a^{3}
$$

By rearranging Equation (2) the semi-major axis is can find as below.

$$
a=\sqrt[3]{M_{*} P^{2}}
$$

Table (1) shows a very well correlation between calculated and observed values of the semi-major axis. In addition, we found 12 unknown values of the semi-major axis in AU (Table 1).

\begin{tabular}{|c|c|c|c|c|c|c|c|c|c|}
\hline \multirow{2}{*}{ No } & \multirow{2}{*}{ Planets Name } & \multirow{2}{*}{ Host Name } & \multirow{2}{*}{$\mathrm{M}_{\mathrm{s}}\left(\mathrm{M}_{\odot}\right)$} & \multirow{2}{*}{$\mathrm{M}_{\mathrm{p}}\left(\mathrm{M}_{\oplus}\right)$} & \multirow{2}{*}{$\mathrm{R}_{\mathrm{p}}\left(\mathrm{R}_{\oplus}\right)$} & \multirow{2}{*}{$\mathrm{P}$ (day) } & \multirow{2}{*}{$a(\mathrm{AU})$} & \multicolumn{2}{|c|}{ Calculated Parameters } \\
\hline & & & & & & & & $\mathrm{P}$ (year) & $a(\mathrm{AU})$ \\
\hline 1 & KOI-55 c & KOI-55 & 0.5 & 0.655 & 0.867 & 0.343 & 0.0076 & 0.0009 & 0.0076 \\
\hline 2 & GJ 1252 b & GJ 1252 & 0.38 & 2.09 & 1.193 & 0.518 & 0.0092 & 0.0014 & 0.0091 \\
\hline 3 & TOI-561 b & TOI-561 & 0.79 & 1.59 & 1.423 & 0.447 & 0.0106 & 0.0012 & 0.0106 \\
\hline 4 & TRAPPIST-1 b & TRAPPIST-1 & 0.08 & 0.85 & 1.086 & 1.511 & 0.0111 & 0.0041 & 0.0111 \\
\hline 5 & Kepler-407 b & Kepler-407 & 1 & 3.2 & 1.07 & 0.669 & - & 0.0018 & 0.0150 \\
\hline 6 & TRAPPIST-1 c & TRAPPIST-1 & 0.08 & 1.38 & 1.056 & 2.422 & 0.0152 & 0.0066 & 0.0152 \\
\hline 7 & GJ 1132 b & GJ 1132 & 0.18 & 1.66 & 1.13 & 1.629 & 0.0153 & 0.0045 & 0.0153 \\
\hline 8 & Kepler-10 b & Kepler-10 & 0.91 & 4.60853 & 1.481 & 0.837 & 0.0172 & 0.0023 & 0.0169 \\
\hline 9 & GJ 486 b & GJ 486 & 0.32 & 2.82 & 1.305 & 1.467 & 0.0173 & 0.0040 & 0.0173 \\
\hline 10 & L $168-9$ b & L 168-9 & 0.62 & 4.6 & 1.39 & 1.402 & 0.0209 & 0.0038 & 0.0209 \\
\hline 11 & L 98-59 b & L 98-59 & 0.31 & 1.01 & 0.8 & 2.253 & 0.0228 & 0.0062 & 0.0228 \\
\hline 12 & $\mathrm{~K} 2-36 \mathrm{~b}$ & $\mathrm{~K} 2-36$ & 0.79 & 3.9 & 1.43 & 1.423 & 0.0223 & 0.0039 & 0.0229 \\
\hline 13 & TOI-178 b & TOI-178 & 0.65 & 1.5 & 1.152 & 1.915 & 0.0261 & 0.0052 & 0.0262 \\
\hline 14 & LHS $1140 \mathrm{c}$ & LHS 1140 & 0.18 & 1.81 & 1.282 & 3.778 & 0.0268 & 0.0104 & 0.0268 \\
\hline 15 & TRAPPIST-1 e & TRAPPIST-1 & 0.08 & 0.62 & 0.918 & 6.100 & 0.0282 & 0.0167 & 0.0282 \\
\hline 16 & L 98-59 c & L 98-59 & 0.31 & 2.42 & 1.35 & 3.690 & 0.0317 & 0.0101 & 0.0316 \\
\hline
\end{tabular}

Table 1 Calculation of Semi-major axis with applying Kepler's Third Law for confirmed Exoplanets. The mass of planets is between 0.5 and $5 M_{\oplus}$ or with radii between 0.8 and $1.5 R_{\oplus}$ for Terran planets. 


$\begin{array}{ll}17 & \text { GJ 357 b } \\ 18 & \text { Kepler-97 b } \\ 19 & \text { Kepler-408 b } \\ 20 & \text { TRAPPIST-1 f } \\ 21 & \text { GJ 9827 c } \\ 22 & \text { TRAPPIST-1 g } \\ 23 & \text { Kepler-406 c } \\ 24 & \text { Kepler-101 c } \\ 25 & \text { HD 21749 c } \\ 26 & \text { Kepler-105 c } \\ 27 & \text { Kepler-107 d } \\ 28 & \text { Kepler-102 d } \\ 29 & \text { Kepler-138 c } \\ 30 & \text { HD 136352 b } \\ 31 & \text { Kepler-595 c } \\ 32 & \text { Kepler-36 b } \\ 33 & \text { Kepler-128 b } \\ 34 & \text { Kepler-138 d } \\ 35 & \text { Kepler-128 c }\end{array}$

$\begin{array}{lc}\text { GJ 357 } & 0.34 \\ \text { Kepler-97 } & 0.94 \\ \text { Kepler-408 } & 1.08 \\ \text { TRAPPIST-1 } & 0.08 \\ \text { GJ 9827 } & 0.61 \\ \text { TRAPPIST-1 } & 0.08 \\ \text { Kepler-406 } & 1.07 \\ \text { Kepler-101 } & 1.17 \\ \text { GJ 143 } & 0.73 \\ \text { Kepler-105 } & 0.96 \\ \text { Kepler-107 } & 1.24 \\ \text { Kepler-102 } & 0.8 \\ \text { Kepler-138 } & 0.52 \\ \text { HD 136352 } & 0.91 \\ \text { Kepler-595 } & 0.93 \\ \text { Kepler-36 } & 1.03 \\ \text { Kepler-128 } & 1.09 \\ \text { Kepler-138 } & 0.52 \\ \text { Kepler-128 } & 1.09\end{array}$

Figure (1), shows $a$ - $P$ relation for 23 confirmed and potentially habitable exoplanets with masses from 0.5 to $5 M_{\oplus}$ with the value of $R^{2}=0.8101$.

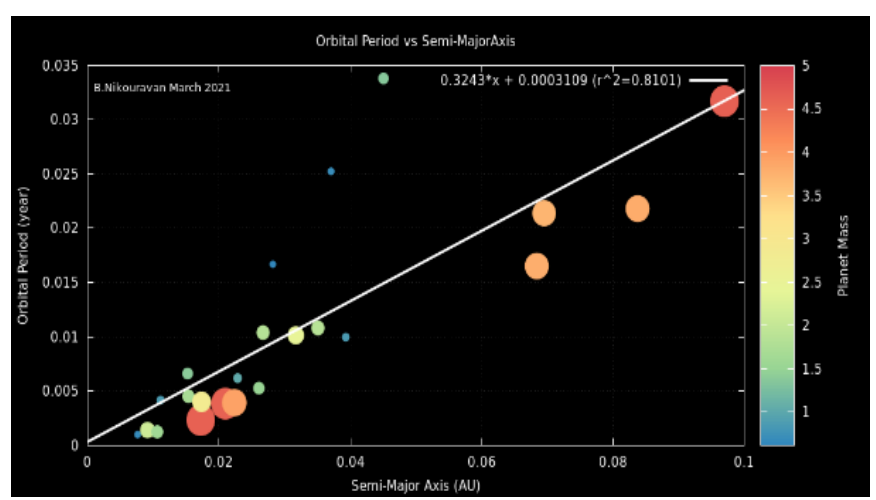

Figure 1 Observational data for orbital Period-Semi major axis of 23 Habitable Exoplanets with $R^{2}=0.8101$.

The probability density function of the semi-major axis for 23 planets has been shown in Figure (3). Exoplanet Histogram of semi-major axis in Kolmogorov Smirnov distribution for 23

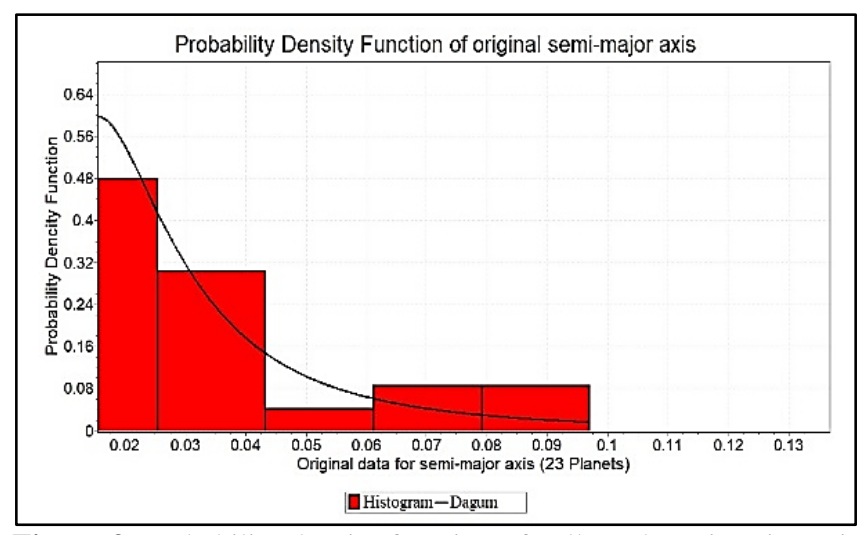

Figure 3 probability density function of collected semi-major axis for 23 Exoplanets - Goodness of Fit (Dagum)
Figure (2), also shows $a-P$ a relationship for calculated 35 confirmed and potentially habitable exoplanets with masses running from 0.5 to $5 M_{\oplus}$ and correlation value $R^{2}=0.9314$.

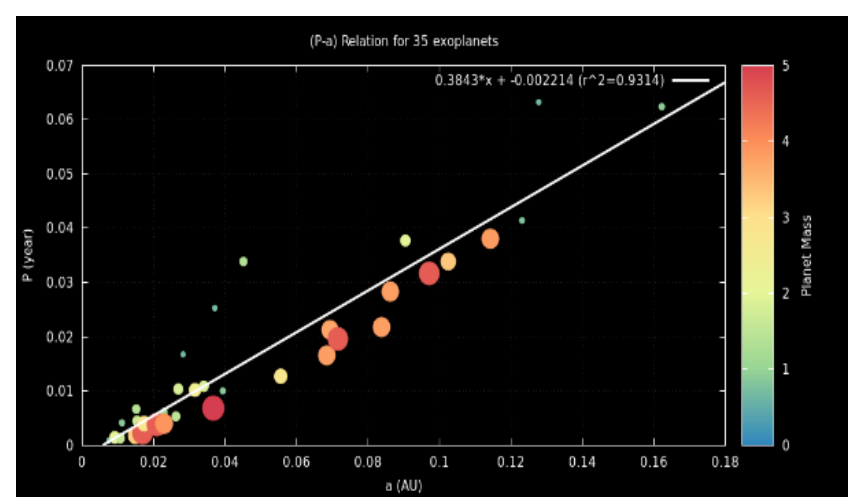

Figure 2 Calculated data for orbital Period-Semi major axis of 35 Habitable Exoplanets with $R^{2}=0.9314$

planets is with the p-value 0.997. Moreover, the probability density function of the semi-major axis Histogram for the calculated data (35 planets), shown in Fig 4.

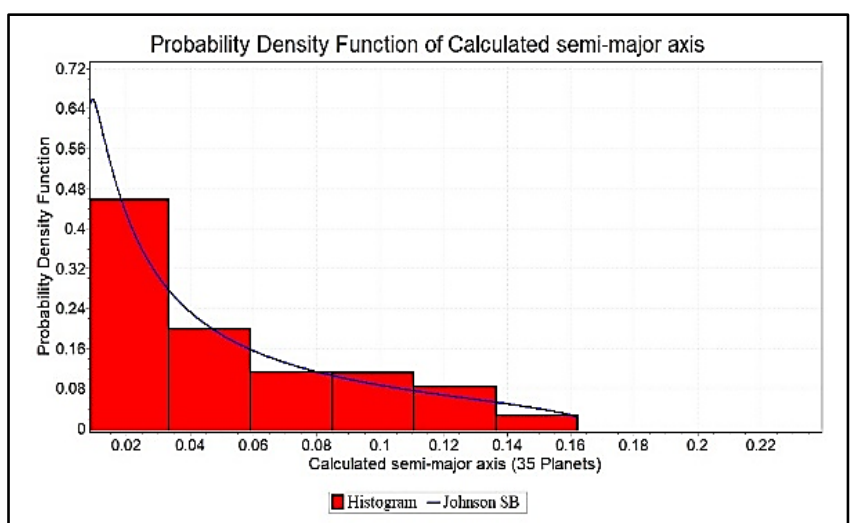

Figure 4 probability density function of calculated semi-major axis for 35 Exoplanets - Goodness of Fit (Jojnson SB) 


\section{MASS CALCULATION}

Using the previous calculation (Semi-major axis of habitable planets) and knowing the mass of the star and orbital period of a planet around the center of mass of its star and combining the Equation (3) and radial velocity amplitude, allows us to work out the mass of the exoplanets. For simplicity, we assume the orbit of the planets is circular and not elliptic. Combine with conservation of momentum law and some unit conversion, gives us the mass of the planet $M_{p}$ in terms of solar mass. With the relationship between the mass of the star, the radial velocity amplitude, the orbital period of the planet, and the semi-major axis of the planet, we obtained the mass of planets as follows.

$$
M_{p}=M_{s}\left(\frac{K P}{2 \pi a}\right)
$$

Here, $M_{S}$ is the mass of a star, $K$ is the radial velocity amplitude and $P$ is the orbital period of the planet in years.
From Equation (4), it is clear that the radial velocity amplitude $K$ and the orbital period of the planet should be known. Therefore, as a result, the mass of the planet in terms of solar mass can be calculated by Equation (5).

$$
M_{p}=\left(2.1 \times 10^{-4}\right) M_{*}\left(\frac{K P}{2 \pi a}\right)
$$

Typically determined masses are around the mass of solar, Jupiter and Earth mass. To convert the mass of the planet from solar mass to Jupiter mass $\left(M_{p(s o l)}\right.$ to $\left.M_{p(j u p)}\right)$, we need to multiply $\mathrm{M}_{\mathrm{p}(\mathrm{sol})}$ by 1047 and to convert from Jupiter mass to Earth mass $M_{p(\text { jup })}$ to $M_{p(E)}$, should multiply by 318 .

Here $M_{p(s o l)}, M_{p(j u p)}$ and $M_{p(E a)}$ are the mass of planets in terms of solar, Jupiter and Earth-mass, respectively. The results of the calculation are shown in Table 2. Here we also inspected the Kepler law relation between star and planet.

\begin{tabular}{|c|c|c|c|c|c|c|c|c|c|c|c|}
\hline \multirow{2}{*}{ No } & \multirow{2}{*}{ Planet Name } & \multirow{2}{*}{ Hostname } & \multirow{2}{*}{$\mathrm{P}($ day $)$} & \multirow{2}{*}{$a(\mathrm{AU})$} & \multirow{2}{*}{$\mathrm{K}(\mathrm{m} / \mathrm{s})$} & \multirow{2}{*}{$\mathrm{M}_{\mathrm{p}}\left(\mathrm{M}_{\oplus}\right)$} & \multirow{2}{*}{$\mathrm{M}_{\mathrm{S}}\left(\mathrm{M}_{\odot}\right)$} & \multicolumn{4}{|c|}{ Calculated Mass in solar, Jupiter, and Earth } \\
\hline & & & & & & & & $a(\mathrm{AU})$ & $\mathrm{M}_{\mathrm{p}}\left(\mathrm{M}_{\odot}\right)$ & $\mathrm{M}_{\mathrm{p}}\left(\mathrm{M}_{\mathrm{j}}\right)$ & $\mathrm{M}_{\mathrm{p}}\left(\mathrm{M}_{\oplus}\right)$ \\
\hline 1 & L 98-59 b & L 98-59 & 2.25 & 0.02282 & 1.06 & 1.01 & 0.31 & 0.0228 & $3.0335 \mathrm{E}-06$ & 0.0032 & 1.01 \\
\hline 2 & Kepler-408 b & Kepler-408 & 2.47 & - & - & 5 & 1.08 & 0.0367 & 1.5017E-05 & 0.0157 & 5.00 \\
\hline 3 & Kepler-406 c & Kepler-406 & 4.62 & - & 1 & 2.71 & 1.07 & 0.0556 & 8.1395E-06 & 0.0085 & 2.71 \\
\hline 4 & Kepler-107 d & Kepler-107 & 7.96 & 0.08377 & 1.06 & 3.8 & 1.24 & 0.0838 & 1.1413E-05 & 0.0119 & 3.80 \\
\hline 5 & KOI-55 c & KOI-55 & 0.34 & 0.0076 & - & 0.655 & 0.5 & 0.0076 & $1.9673 \mathrm{E}-06$ & 0.0021 & 0.66 \\
\hline 6 & HD 21749 c & GJ 143 & 7.79 & 0.0695 & 1.43 & 3.7 & 0.73 & 0.0693 & $1.1113 \mathrm{E}-05$ & 0.0116 & 3.70 \\
\hline 7 & TRAPPIST-1 e & TRAPPIST-1 & 6.10 & 0.02817 & - & 0.62 & 0.08 & 0.0282 & $1.8622 \mathrm{E}-06$ & 0.0019 & 0.62 \\
\hline 8 & Kepler-595 c & Kepler-595 & 12.39 & - & - & 3.3 & 0.93 & 0.1023 & 9.9115E-06 & 0.0104 & 3.30 \\
\hline 9 & TRAPPIST-1 f & TRAPPIST-1 & 9.21 & 0.0371 & - & 0.68 & 0.08 & 0.0371 & 2.0424E-06 & 0.0021 & 0.68 \\
\hline 10 & TRAPPIST-1 c & TRAPPIST-1 & 2.42 & 0.01521 & - & 1.38 & 0.08 & 0.0152 & 4.1448E-06 & 0.0043 & 1.38 \\
\hline 11 & Kepler-407 b & Kepler-407 & 0.67 & - & - & 3.2 & 1 & 0.0150 & $9.6112 \mathrm{E}-06$ & 0.0101 & 3.20 \\
\hline 12 & TRAPPIST-1 b & TRAPPIST-1 & 1.5 & 0.01111 & - & 0.85 & 0.08 & 0.0111 & $2.5530 \mathrm{E}-06$ & 0.0027 & 0.85 \\
\hline 13 & TRAPPIST-1 g & TRAPPIST-1 & 12.35 & 0.0451 & - & 1.34 & 0.08 & & 4.0247E-06 & 0.0042 & 1.34 \\
\hline 14 & GJ 1132 b & GJ 11 & & 0.0153 & 2.85 & 1.66 & 0.18 & & 4.9858E-06 & & 1.66 \\
\hline 15 & TOI-178 b & TOI- 1 & & 0.02607 & 1.05 & 1.5 & 0.65 & & 4.5052E-06 & 0.0047 & 1.50 \\
\hline 16 & Kepler-102 d & Keple & 10. & - & 1.37 & & & & $1.1413 \mathrm{E}-05$ & 0.0119 & 3.80 \\
\hline 17 & GJ 1252 b & GJ 1 & & 0.00916 & 3.17 & 2.09 & 0.38 & & $6.2773 \mathrm{E}-06$ & & 2.09 \\
\hline 18 & $r-138 c$ & & 13. & - & - & 1.97 & 0.52 & & $5.9169 \mathrm{E}-06$ & 062 & 1.97 \\
\hline 19 & $-138 d$ & -138 & 23.09 & - & - & 0.64 & 0.52 & 0.1277 & $1.9222 \mathrm{E}-06$ & 0.0020 & 0.64 \\
\hline 20 & GJ 357 b & & 3.93 & 0.035 & 1.52 & 1.84 & 0.34 & 0.0340 & $5.5264 \mathrm{E}-06$ & 0.0058 & 1.84 \\
\hline 21 & GJ 9827 c & GJ 9827 & 3.65 & 0.03925 & 0.49 & 0.84 & 0.61 & 0.0394 & $2.5229 \mathrm{E}-06$ & 0.0026 & 0.84 \\
\hline 22 & Kepler-101 c & Kepler-101 & 6.03 & 0.0684 & 1.17 & 3.78 & 1.17 & 0.0683 & $1.1353 \mathrm{E}-05$ & 0.0119 & 3.78 \\
\hline 23 & LHS 1140 c & LHS 1140 & 3.78 & 0.02675 & 2.35 & 1.81 & 0.18 & 0.0268 & 5.4363E-06 & 0.0057 & 1.81 \\
\hline 24 & GJ 486 b & GJ 486 & 1.47 & 0.01734 & 3.37 & 2.82 & 0.32 & 0.0173 & 8.4698E-06 & 0.0089 & 2.82 \\
\hline 25 & Kepler-105 c & Kepler-105 & 7.13 & - & - & 4.6 & 0.96 & 0.0715 & $1.3816 \mathrm{E}-05$ & 0.0145 & 4.60 \\
\hline 26 & Kepler-128 c & Kepler-128 & 22.80 & - & - & 0.9 & 1.09 & 0.1620 & 2.7031E-06 & 0.0028 & 0.90 \\
\hline 27 & L $98-59$ c & L 98-59 & 3.69 & 0.0317 & 2.21 & 2.42 & 0.31 & 0.0316 & $7.2684 \mathrm{E}-06$ & 0.0076 & 2.42 \\
\hline 28 & L $168-9$ b & L $168-9$ & 1.40 & 0.02091 & 3.66 & 4.6 & 0.62 & 0.0209 & $1.3816 \mathrm{E}-05$ & 0.0145 & 4.60 \\
\hline 29 & TOI-561 b & TOI-561 & 0.45 & 0.01055 & 1.56 & 1.59 & 0.79 & 0.0106 & 4.7755E-06 & 0.0050 & 1.59 \\
\hline 30 & $\mathrm{~K} 2-36 \mathrm{~b}$ & $\mathrm{~K} 2-36$ & 1.42 & 0.0223 & 2.6 & 3.9 & 0.79 & 0.0229 & $1.1714 \mathrm{E}-05$ & 0.0123 & 3.90 \\
\hline 31 & Kepler-128 b & Kepler-128 & 15.09 & - & - & 0.77 & 1.09 & 0.1230 & $2.3127 \mathrm{E}-06$ & 0.0024 & 0.77 \\
\hline 32 & Kepler-97 b & Kepler-97 & 2.59 & - & 1.65 & 3.51 & 0.94 & 0.0361 & $1.0542 \mathrm{E}-05$ & 0.0110 & 3.51 \\
\hline 33 & Kepler-10 b & Kepler-10 & 0.84 & 0.0172 & 3.3 & 4.60853 & 0.91 & 0.0169 & $1.3842 \mathrm{E}-05$ & 0.0145 & 4.61 \\
\hline 34 & HD $136352 \mathrm{~b}$ & & 11.58 & 0.0969 & 1.4 & 4.62 & 0.91 & 0.0971 & $1.3876 \mathrm{E}-05$ & 0.0145 & 4.62 \\
\hline 35 & Kepler-36 b & Kepler-36 & 13.87 & - & - & 3.83 & 1.03 & 0.1141 & $1.1503 \mathrm{E}-05$ & 0.0120 & 3.83 \\
\hline
\end{tabular}

Table 2 Calculation of mass of exoplanets in the Solar, Jupiter, and Earth-mass. 


\section{STELLAR LUMINOSITY OF STAR $\left(\mathrm{L}_{\odot}\right)$}

Another important physical quantity of brightness is luminosity. It is the power of a star the amount of energy that a star emits from its surface. A star's luminosity can be determined from two stellar characteristics, radius and effective temperature of the star. To determine a star's radius, we need two other metrics. The first one is the star's angular diameter and the second one is the distance of the star from Earth. Since the effective temperature is merely a number that represents the temperature of a black body that would reproduce the luminosity, it obviously cannot be measured directly, but it can be estimated from the spectrum.
But here we determine the stellar luminosity of the star in terms of radius and temperature of the star $(R T)$. Stellar luminosity in solar units given the star radius $R_{S}$ in solar units and temperature $T_{s}$ in kelvins.

$$
\frac{L_{S}}{L_{\odot}}=\left(\frac{R_{S}}{R_{\odot}}\right)^{2}\left(\frac{T_{S}}{T_{\odot}}\right)^{4}
$$

Here we use the $L_{\odot}=3.846 \times 10^{26}$ in watt, and $T_{\odot}=$ 5780 in kelvin and $R_{\odot}=6.955 \times 10^{8}$ in meter. The estimated values for the luminosity $L_{s}$ are shown in Table 3.

Table 3 Determination of Stellar Luminosity of star in terms of Radius and Temperature of star

\begin{tabular}{|c|c|c|c|c|c|c|c|}
\hline \multirow{2}{*}{ No } & \multirow{2}{*}{ Planets } & \multirow{2}{*}{ Host } & \multirow{2}{*}{$T_{\text {eff }}$} & \multirow{2}{*}{$R_{\odot}$} & \multirow{2}{*}{$L_{s}$} & \multicolumn{2}{|c|}{ Calculated } \\
\hline & & & & & & $L_{S}$ & $L_{S} \log 10\left(L_{\odot}\right)$ \\
\hline 1 & KOI-55 c & KOI-55 & 27730 & 0.2 & 1.36 & 21.19083725 & 1.326 \\
\hline 2 & GJ 1252 b & GJ 1252 & 3458 & 0.39 & -1.708 & 0.019485781 & -1.710 \\
\hline 3 & TOI-561 b & TOI-561 & 5455 & 0.85 & - & 0.573199123 & -0.242 \\
\hline 4 & TRAPPIST- $1 \mathrm{~b}$ & TRAPPIST-1 & 2559 & 0.12 & -3.281 & 0.000553264 & -3.257 \\
\hline 5 & Kepler-407 b & Kepler-407 & 5476 & 1.01 & - & 0.821835982 & -0.085 \\
\hline 6 & TRAPPIST-1 c & TRAPPIST-1 & 2559 & 0.12 & -3.281 & 0.000553264 & -3.257 \\
\hline 7 & GJ 1132 b & GJ 1132 & 3270 & 0.21 & -2.359 & 0.004517709 & -2.345 \\
\hline 8 & Kepler-10 b & Kepler-10 & 5627 & 1.06 & - & 1.009271564 & 0.004 \\
\hline 9 & GJ 486 b & GJ 486 & 3340 & 0.33 & -1.917 & 0.01214234 & -1.916 \\
\hline 10 & L $168-9 b$ & L 168-9 & 3800 & 0.6 & -1.172 & 0.067255148 & -1.172 \\
\hline 11 & L 98-59 b & L 98-59 & 3412 & 0.31 & - & 0.011669389 & -1.933 \\
\hline 12 & $\mathrm{~K} 2-36 \mathrm{~b}$ & $\mathrm{~K} 2-36$ & 4916 & 0.72 & -0.57 & 0.271269606 & -0.567 \\
\hline 13 & TOI-178 b & TOI-178 & 4316 & 0.65 & -0.879 & 0.131353335 & -0.882 \\
\hline 14 & LHS 1140 c & LHS 1140 & 3216 & 0.21 & -2.356 & 0.004226602 & -2.374 \\
\hline 16 & L 98-59 c & L 98-59 & 3412 & 0.31 & - & 0.011669389 & -1.933 \\
\hline 17 & GJ 357 b & GJ 357 & 3505 & 0.34 & -1.798 & 0.015631425 & -1.806 \\
\hline 18 & Kepler-97 b & Kepler-97 & 5779 & 0.98 & - & 0.959735536 & -0.018 \\
\hline 19 & Kepler-408 b & Kepler-408 & 6104 & 1.23 & - & 1.881728528 & 0.275 \\
\hline 20 & TRAPPIST- $1 \mathrm{f}$ & TRAPPIST-1 & 2559 & 0.12 & -3.281 & 0.000553264 & -3.257 \\
\hline 21 & GJ 9827 c & GJ 9827 & 4340 & 0.6 & - & 0.114432679 & -0.941 \\
\hline 22 & TRAPPIST- $1 \mathrm{~g}$ & TRAPPIST-1 & 2559 & 0.12 & -3.281 & 0.000553264 & -3.257 \\
\hline 23 & Kepler-406 c & Kepler-406 & 5538 & 1.07 & - & 0.964868231 & -0.016 \\
\hline 24 & Kepler-101 c & Kepler-101 & 5667 & 1.56 & - & 2.24879928 & 0.352 \\
\hline 25 & HD 21749 c & GJ 143 & 4640 & 0.69 & -0.686 & 0.19772371 & -0.704 \\
\hline 26 & Kepler-105 c & Kepler-105 & 5827 & 0.89 & - & 0.81817976 & -0.087 \\
\hline 27 & Kepler-107 d & Kepler-107 & 5854 & 1.45 & - & 2.212256721 & 0.345 \\
\hline 28 & Kepler-102 d & Kepler-102 & 4903 & 0.74 & - & 0.283530418 & -0.547 \\
\hline 29 & Kepler-138 c & Kepler-138 & 3841 & 0.44 & - & 0.037754718 & -1.423 \\
\hline 30 & HD $136352 b$ & HD 136352 & 5850 & 1.01 & 0.034 & 1.070421585 & 0.030 \\
\hline 33 & Kepler-128 b & Kepler-128 & - & 1.61 & - & - & - \\
\hline 34 & Kepler-138 d & Kepler-138 & 3841 & 0.44 & - & 0.037754718 & -1.423 \\
\hline 35 & Kepler-128 c & Kepler-128 & - & 1.61 & - & - & - \\
\hline
\end{tabular}

\section{CALCULATE HABITABILITY}

The Habitable Zone (HZ) simply is the range of orbits around a star on which a planetary surface can support liquid water given sufficient atmospheric pressure. The inner and outer boundaries are defined from Venus to Mars models, respectively, from (Kasting, Whitmire, \& Reynolds, 1993). For a Sun-like star (G2), these are given at 0.75 and 1.77AU.
To estimate HZ, we neglect the stellar effective temperature and define the center of $\mathrm{HZ}$ as $1 \mathrm{AU}$ for Earth around the Sun, and also scale with stellar luminosity. According to Equations 8,9 , and 10 , and using the luminosity of stars $L_{s}$ and the luminosity of the sun (as a constant), it is possible to calculate the inner, center, and outer regions of the Habitability Zone for exoplanets (NASA Exoplanet Archive, 2021). 


$$
\begin{gathered}
R_{H Z(\text { in })}(A U)=0.75 \sqrt{\frac{L_{s}}{L_{\odot}}} \\
R_{H Z(\text { cent })}(A U)=1.0 \sqrt{\frac{L_{S}}{L_{\odot}}} \\
R_{H Z(\text { out })}(A U)=1.77 \sqrt{\frac{L_{S}}{L_{\odot}}}
\end{gathered}
$$

$$
\Delta(H Z)=R_{H Z(\text { out })}-R_{H Z(\text { in })}
$$

By applying these equations to determine the habitability zone of 3522 planets, out of 3945 approved exoplanets were used. Finally, habitability zone was calculated for Inner, Center,

\begin{tabular}{|c|c|c|c|c|c|c|c|}
\hline \multirow{2}{*}{ No } & \multirow{2}{*}{ Planet Name } & \multirow{2}{*}{ Star Name } & \multirow{2}{*}{ Ls } & \multicolumn{4}{|c|}{ Calculated Habitability Zone } \\
\hline & & & & $\mathrm{Hz}$ (Inner) & $\mathrm{Hz}$ (center) & $\mathrm{Hz}$ (Outer) & $\Delta(H Z)$ \\
\hline 1 & HD $81817 \mathrm{~b}$ & HD 81817 & 3.267 & 32.24 & 42.99 & 76.10 & 43.85 \\
\hline 2 & $\mathrm{BD}+202457 \mathrm{~b}$ & BD+20 2457 & 3.172 & 28.92 & 38.56 & 68.25 & 39.33 \\
\hline 3 & HD $240237 \mathrm{~b}$ & HD 240237 & 3.033 & 24.65 & 32.86 & 58.17 & 33.52 \\
\hline 4 & $\mathrm{BD}+202457 \mathrm{c}$ & BD+20 2457 & 2.799 & 18.83 & 25.10 & 44.43 & 25.60 \\
\hline 5 & HD 158996 b & HD 158996 & 2.793 & 18.70 & 24.93 & 44.12 & 25.43 \\
\hline 6 & HD 208527 b & HD 208527 & 2.793 & 18.68 & 24.90 & 44.08 & 25.40 \\
\hline 7 & HD 96127 b & HD 96127 & 2.752 & 17.84 & 23.78 & 42.09 & 24.26 \\
\hline 8 & HD $220074 b$ & HD 220074 & 2.745 & 17.68 & 23.58 & 41.73 & 24.05 \\
\hline 9 & HD $95127 \mathrm{~b}$ & HD 95127 & 2.678 & 16.38 & 21.84 & 38.66 & 22.28 \\
\hline 10 & NGC 2682 Sand 364 b & NGC 2682 Sand 364 & 2.675 & 16.31 & 21.75 & 38.49 & 22.18 \\
\hline 11 & HD $4760 \mathrm{~b}$ & HD 4760 & 2.648 & 15.81 & 21.09 & 37.32 & 21.51 \\
\hline 12 & HD $112640 \mathrm{~b}$ & HD 112640 & 2.609 & 15.12 & 20.15 & 35.67 & 20.56 \\
\hline 13 & HD 13189 b & HD 13189 & 2.604 & 15.03 & 20.04 & 35.47 & 20.44 \\
\hline 14 & HD $24064 \mathrm{~b}$ & HD 24064 & 2.587 & 14.74 & 19.66 & 34.79 & 20.05 \\
\hline 16 & $\mathrm{BD}+032562 \mathrm{~b}$ & $\mathrm{BD}+032562$ & 2.421 & 12.18 & 16.24 & 28.74 & 16.56 \\
\hline 17 & 11 UMi b & $11 \mathrm{UMi}$ & 2.399 & 11.87 & 15.83 & 28.01 & 16.14 \\
\hline 18 & tau Gem b & tau Gem & 2.378 & 11.58 & 15.45 & 27.34 & 15.75 \\
\hline 19 & HD 60292 b & HD 60292 & 2.368 & 11.46 & 15.28 & 27.04 & 15.58 \\
\hline 20 & HD $47536 \mathrm{~b}$ & HD 47536 & 2.259 & 10.11 & 13.48 & 23.85 & 13.75 \\
\hline 21 & HD 1690 b & HD 1690 & 2.187 & 9.30 & 12.40 & 21.96 & 12.65 \\
\hline 22 & HD $110014 b$ & HD 110014 & 2.184 & 9.27 & 12.36 & 21.88 & 12.61 \\
\hline 23 & eps $\mathrm{CrB}$ b & eps $\mathrm{CrB}$ & 2.173 & 9.15 & 12.20 & 21.60 & 12.45 \\
\hline 24 & HD $66141 b$ & HD 66141 & 2.156 & 8.98 & 11.97 & 21.19 & 12.21 \\
\hline 25 & BD-13 $2130 b$ & BD-13 2130 & 2.148 & 8.89 & 11.85 & 20.98 & 12.09 \\
\hline 26 & HD 11755 b & HD 11755 & 2.118 & 8.59 & 11.45 & 20.27 & 11.68 \\
\hline 27 & $4 \mathrm{UMa}$ b & $4 \mathrm{UMa}$ & 2.048 & 7.92 & 10.57 & 18.70 & 10.78 \\
\hline 28 & nu Oph c & nu Oph & 2.037 & 7.82 & 10.43 & 18.47 & 10.64 \\
\hline 29 & nu Oph b & nu Oph & 2.037 & 7.82 & 10.43 & 18.47 & 10.64 \\
\hline 30 & $\mathrm{BD}+152940 \mathrm{~b}$ & $\mathrm{BD}+152940$ & 2.010 & 7.59 & 10.12 & 17.91 & 10.32 \\
\hline 33 & TYC 4282-00605-1 b & TYC 4282-00605-1 & 1.906 & 6.73 & 8.97 & 15.88 & 9.15 \\
\hline 34 & HD 173416 b & HD 173416 & 1.895 & 6.65 & 8.86 & 15.69 & 9.04 \\
\hline 35 & HD 81688 b & HD 81688 & 1.888 & 6.59 & 8.79 & 15.56 & 8.97 \\
\hline 36 & HD 238914 b & HD 238914 & 1.876 & 6.50 & 8.67 & 15.34 & 8.84 \\
\hline 37 & alf Ari b & alf Ari & 1.872 & 6.47 & 8.62 & 15.27 & 8.80 \\
\hline 38 & HD 14067 b & HD 14067 & 1.870 & 6.45 & 8.61 & 15.23 & 8.78 \\
\hline 39 & HIP 107773 b & HIP 107773 & 1.858 & 6.37 & 8.49 & 15.03 & 8.66 \\
\hline 40 & xi Aql b & xi Aql & 1.828 & 6.16 & 8.21 & 14.53 & 8.37 \\
\hline
\end{tabular}
Outer and $\Delta(H Z)$ region. (Table 4 shows only 40 exoplanets).

Table 4 Calculated values of HZ for 3522 approved exoplanets in the Inner, center, and outer region. Here we show only a small part of the result of the calculation.

\section{RADIAL VELOCITY AMPLITUDE (RVA)}

The radial velocity represents the speed of the object which moves away (positive sign) and /or approaches (negative sign) from Earth. Here we calculate the radial velocity (RVA) amplitude $K$, of each exoplanet with its estimated mass. The predicted radial velocity amplitude is, depends on the planet orbital period, planet mass, and stellar mass. Using the following expression, we find $K$ values in the SI unit.

$$
K=\left(\frac{M_{p}}{M_{S}}\right)\left(\frac{a}{P}\right)\left(\frac{2 \pi}{69.91866}\right)
$$

Where the $M_{p}$ and $M_{s}$ are the mass of the planet and its host respectively, $a$ is the semi-major axis of the planet and $P$ is the planet orbital period. Table 4 shows calculated values for $K$, in $\mathrm{m} / \mathrm{s}$ unit. 
Table 5 Radial velocity amplitude $K$, of each exoplanet

\begin{tabular}{|c|c|c|c|c|}
\hline \multirow{2}{*}{ No } & \multirow{2}{*}{ Planet } & \multirow{2}{*}{$M_{S}\left(M_{\odot}\right)$} & \multicolumn{2}{|c|}{ Calculated Radial velocity amplitude } \\
\hline & & & $P($ year $)$ & $K(\mathrm{~m} / \mathrm{s})$ \\
\hline 1 & TRAPPIST-1 e & 0.08 & 0.0167 & 1.17 \\
\hline 2 & TRAPPIST-1 f & 0.08 & 0.0252 & 1.12 \\
\hline 3 & TRAPPIST-1 c & 0.08 & 0.0066 & 3.55 \\
\hline 4 & TRAPPIST- $1 \mathrm{~b}$ & 0.08 & 0.0041 & 2.56 \\
\hline 5 & TRAPPIST-1 g & 0.08 & 0.0338 & 2.00 \\
\hline 6 & TOI-178 b & 0.65 & 0.0052 & 1.03 \\
\hline 7 & Kepler-102 d & 0.8 & 0.0283 & 1.30 \\
\hline 8 & TOI-561 b & 0.79 & 0.0012 & 1.56 \\
\hline 9 & Kepler-107 d & 1.24 & 0.0218 & 1.06 \\
\hline 10 & L 98-59 b & 0.31 & 0.0062 & 1.08 \\
\hline 11 & Kepler-138 c & 0.52 & 0.0378 & 0.82 \\
\hline 12 & Kepler-138 d & 0.52 & 0.0633 & 0.22 \\
\hline 13 & GJ 357 b & 0.34 & 0.0108 & 1.54 \\
\hline 14 & GJ $9827 \mathrm{c}$ & 0.61 & 0.0100 & 0.49 \\
\hline 15 & L 98-59 c & 0.31 & 0.0101 & 2.19 \\
\hline 16 & HD $136352 b$ & 0.91 & 0.0317 & 1.40 \\
\hline 17 & Kepler-406 c & 1.07 & 0.0127 & 1.00 \\
\hline 18 & KOI-55 c & 0.5 & 0.0009 & 0.95 \\
\hline 19 & HD $21749 c$ & 0.73 & 0.0213 & 1.48 \\
\hline 20 & Kepler-595 c & 0.93 & 0.0339 & 0.96 \\
\hline 21 & Kepler-407 b & 1 & 0.0018 & 2.35 \\
\hline 22 & GJ 1132 b & 0.18 & 0.0045 & 2.84 \\
\hline 23 & Kepler-101 c & 1.17 & 0.0165 & 1.20 \\
\hline 24 & LHS $1140 \mathrm{c}$ & 0.18 & 0.0104 & 2.34 \\
\hline 25 & Kepler-105 c & 0.96 & 0.0195 & 1.58 \\
\hline 26 & Kepler-128 c & 1.09 & 0.0625 & 0.19 \\
\hline 27 & $\mathrm{~K} 2-36 \mathrm{~b}$ & 0.79 & 0.0039 & 2.60 \\
\hline 28 & Kepler-128 b & 1.09 & 0.0413 & 0.19 \\
\hline 29 & Kepler-97 b & 0.94 & 0.0071 & 1.71 \\
\hline 30 & Kepler-10 b & 0.91 & 0.0023 & 3.34 \\
\hline 31 & Kepler-36 b & 1.03 & 0.0380 & 1.00 \\
\hline 32 & Kepler-408 b & 1.08 & 0.0068 & 2.26 \\
\hline 33 & GJ 1252 b & 0.38 & 0.0014 & 3.18 \\
\hline 34 & GJ 486 b & 0.32 & 0.0040 & 3.41 \\
\hline 35 & L $168-9 \mathrm{~b}$ & 0.62 & 0.0038 & 3.63 \\
\hline
\end{tabular}

\section{EQUILIBRIUM TEMPERATURES OF PLANETS}

The planetary equilibrium temperature (PET) or effective radiation emission temperature, is a theoretical temperature that a planet would be a black body being heated only by its parent star and it can be determined by the size, temperature, and distance to its parent star, but not on its size (Stull \& Ahrens, 2015, p. 794).

PET is different from the global mean temperature and surface air temperature, which is measured observationally by satellites or surface-based instruments, and may be warmer than an equilibrium temperature due to greenhouse effects.

When the planet orbiting its host star then it can collect some fraction of radiation emitted from the star. The planet albedo is depending on the characteristics of its surface and atmosphere, and then only absorbs a fraction of radiation and therefore the planet receives a certain amount of energy from the star and heats up which it re-radiates as a blackbody. Herewith using this information, we aim to calculate the equilibrium temperature of the planet. Let's start with figuring out how much energy per unit of time a planet receives.
The planet has a cross-sectional area of $\pi R^{2}$ (and not $4 \pi R^{2}$ ), so the fraction of all the energy the star emitted and the planet receives is that cross-sectional area, divided by the surface area of the sphere out to the planet.

$$
\left(\frac{\pi R^{2}}{4 \pi d^{2}}\right) \rightarrow\left(\frac{R}{2 d}\right)^{2}
$$

the luminosity of the star is also defined by,

$$
L_{\odot}=4 \pi R_{\odot}^{2} \sigma T_{\odot}^{4}
$$

Therefore, the total amount of energy per unit of time received by the planet is

$$
L_{\odot}\left(\frac{R_{p}}{2 d}\right)^{2}
$$

or

$$
E=\left(4 \pi R_{\odot}^{2} \sigma T_{\odot}^{4}\right)\left(\frac{R_{p}}{2 d}\right)^{2}
$$


This is correct if the planet absorbs all the energy, but it is not correct because some of the energy is reflected outward. Here we need to introduce a new parameter as Albedo $A$ as the fraction of energy per unit time that is reflected by the planet. We note that $A$ is a physical property with no dimension and only is a number. Therefore,

$$
(1-A)
$$

This is the amount of energy absorbed by the planet. A planet completely covered with snow or ice would have an albedo close to $100 \%$, while a completely dark planet would have an albedo close to zero. Thus, the total amount of energy per unit of time absorbed by the planet is

$$
(1-A) E=(1-A)\left[\left(4 \pi R_{\odot}^{2} \sigma T_{\odot}^{4}\right)\left(\frac{R_{p}}{2 d}\right)^{2}\right]
$$

This energy is radiated by the planet in the form of blackbody radiation. Now if the planet has a temperature $T_{p}$ then the amount of energy it is putting out per unit of time is

$$
L_{p}=\left(4 \pi R_{p}^{2}\right) \sigma T_{p}^{4}
$$

If the planet is in equilibrium, then the amount of energy it is absorbing is in the following form.

$$
\left(4 \pi R_{p}^{2}\right) \sigma T_{p}^{4}=(1-A)\left[\left(4 \pi R_{\odot}^{2} \sigma T_{\odot}^{4}\right)\left(\frac{R_{p}}{2 d}\right)^{2}\right](
$$

After simplification the above Equation is

$$
T_{p}^{4}=(1-A) T_{\odot}^{4}\left(\frac{R_{\odot}}{2 d}\right)^{2}
$$

or

$$
T_{p}=T_{\odot}(1-A)^{1 / 4}\left(\frac{R_{\odot}}{2 d}\right)^{\frac{1}{2}}
$$

Equation (22) shows that EPT is determined by the size, temperature, and distance to its parent star but not by its size. For example, the value of PTE for the Earth can be calculated as PTE $=260 K^{o}(-13 \mathrm{C})$ when, $T_{\odot}=5780 \mathrm{~K}, A=40 \%$, $R_{\odot}=7 \times 10^{5} / \mathrm{km}$ and $d=1.5 \times 10^{8} / \mathrm{km}=1$ AU.

This temperature is below the freezing point of water, and therefore, oceans would be largely frozen and it is not true. So, what is missing here in the calculation is another effect, knowing as the Greenhouse Effect. The Greenhouse Effect trapping the heat radiation from the Earth's surface, which raises the mean temperature at the Earth's surface to $300 \mathrm{~K}$.

\section{PLANET DENSITY}

One of the most important properties of planets is their density. The amount of planet density tells us what a planet is made of and how much material it has in space. This property simply can be calculated with the mass and radius of the planet. Normally planets are in a different wide range of sizes and masses but planets made of the same material will have the same density regardless of their size and mass.

Since planets are roughly spherical, calculate the volume of a sphere using the radius. Then divide the mass by the volume of the sphere to get the density.

$$
\rho=\frac{M_{p}}{V}=\frac{M_{p}}{\left(\frac{4}{3} \pi R_{p}^{3}\right)}
$$

A huge and massive planet can have the same density as a small, low-mass planet if they are made of the same material.

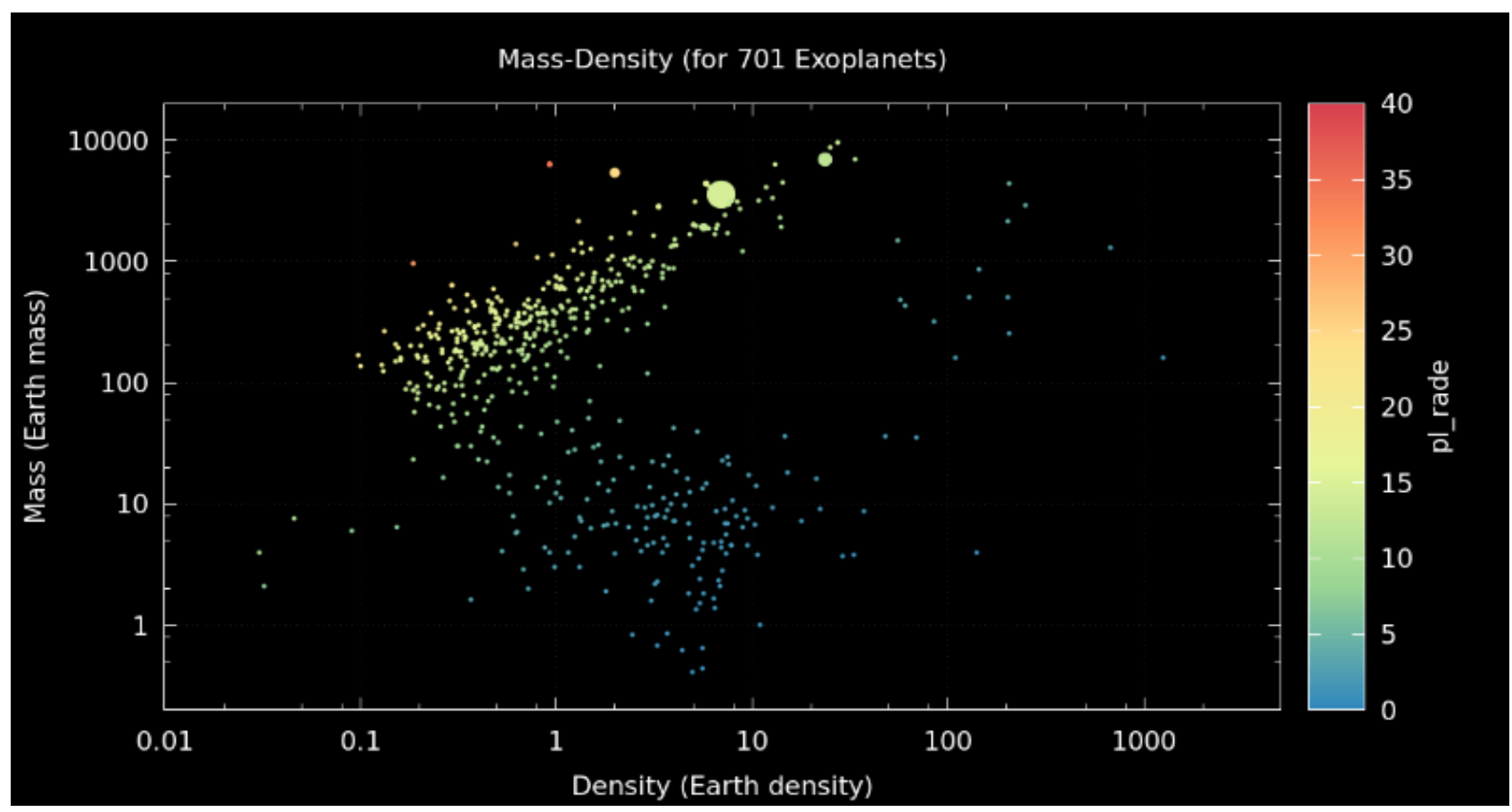

Figure 5 Mass density relation for 701 exoplanets 
Terrestrial planets (Earth-like) have a rocky structure with silicate rocks on the surface and densities between 4 to 5 relatives to the density of water with silicate rocks on the surface. Silicate rock has a density of 3 , less than the average density of a terrestrial planet, and iron has a density of 7.8, more than the average density of a terrestrial planet. Therefore, terrestrial planets have average densities greater than that for the silicate rocks on their surface, they must have denser material under the surface to make the overall average density what it is. Iron and nickel are present in meteorites and the presence of magnetic fields in some of the terrestrial planets shows that they have cores of iron and nickel.

As we know, the magnetic fields can be produced by the motion of liquid iron and nickel. Therefore, according to these facts, the terrestrial planets are made of silicate rock surrounding an iron-nickel core. We remember that the density of Earth is $5.52 \mathrm{in} \mathrm{gr} / \mathrm{cm}^{3}$.

\section{EXOPLANETS CATALOGUING PHL}

A potentially habitable exoplanet is a theoretical type of planet that has liquid water and may support life (Planetary Habitability Laboratory, 2021). An exoplanet would have to have a mass in the range of $\left(0.1 \leq \mathrm{M}_{\mathrm{p}} \leq 10\right) \mathrm{M}_{\oplus}$ (Raymond, Quinn, \& Lunine, 2007) and its radius would be in the range of $(0.4 \leq R \leq 2.5) R_{\oplus}$ (Alibert \& Astrophysics, 2014). Accordingly, one Sub-Terran (Mars-size), 23 Terran (Earthsize), and 36 Super Terran (Super-Earths) founded by end of Oct 2020 and introduced in $\boldsymbol{P H} \boldsymbol{L}^{1}$ (see Table 6).

In comparison with Earth, exoplanets may be smaller, similar, and/or larger than the Earth in mass and radius. Updated this catalog from 2020 to the end of April 2021 shows in Table 6 from 60 exoplanets to 76 . The result shows a total of 76 exoplanets with 2 Subterranean, 36 Terran, and 38 Superterran (Table 6). The results are shown in Figures 8, 9, and 10 below.

Table 6 Classification of potentially habitable Exoplanets with masses in the range of $\left(0.1 \leq M_{p} \leq 10\right) M_{\oplus}$ and radius $(0.4 \leq \mathrm{R} \leq 2.5) \mathrm{R}_{\oplus}$

\begin{tabular}{|c|c|c|c|c|}
\hline Exoplanets Types & $M\left(M_{\oplus}\right)$ & $R\left(R_{\oplus}\right)$ & $2020 *$ & $\begin{array}{c}2021^{* *} \\
\left(0.1 \leq \mathrm{M}_{\mathrm{p}} \leq 10\right) \mathrm{M}_{\oplus}\end{array}$ \\
\hline Subterran (Mars-size) & $0.1-0.5$ & $0.4-0.8$ & 1 & 2 \\
\hline Terran (Earth-size) & $0.5-5$ & $0.8-1.5$ & 23 & 36 \\
\hline \multirow[t]{2}{*}{ Superterran (Super-Earths) } & $5-10$ & $1.5-2.5$ & 36 & 38 \\
\hline & & & 60 & 76 \\
\hline
\end{tabular}

* http://phl.upr.edu/projects/habitable-exoplanets-catalog

** Data collected from NASA Exoplanets Archive April 2021

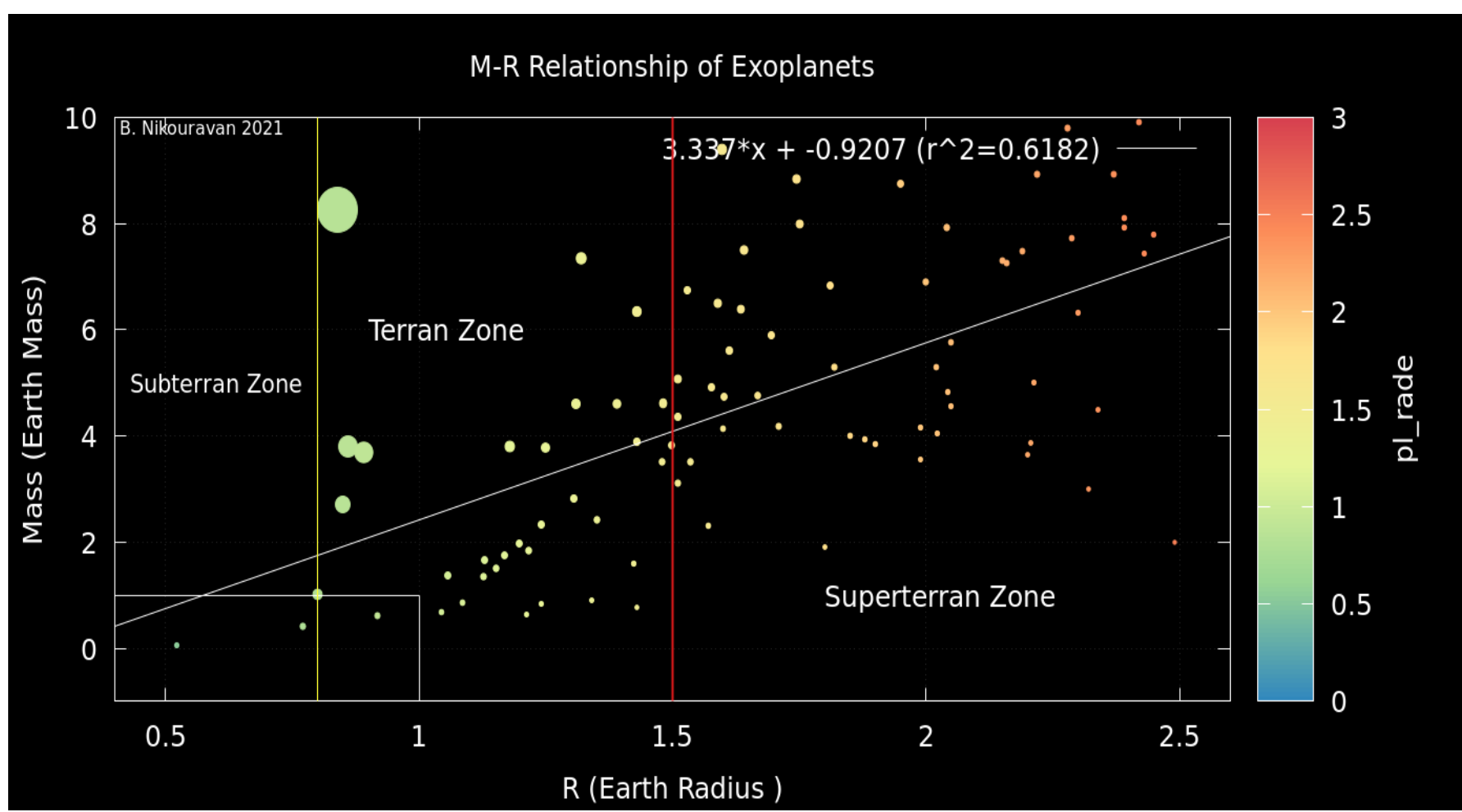

Figure 6 The M-R Relationship of 118 confirmed and habitable exoplanets. The mass and radius are $\left(0.1 \leq M_{p} \leq 10\right) M_{\oplus}$ and $(0.4 \leq R \leq 2.5) R_{\oplus}$.

${ }^{1}$ http://phl.upr.edu/projects/habitable-exoplanets-catalog 


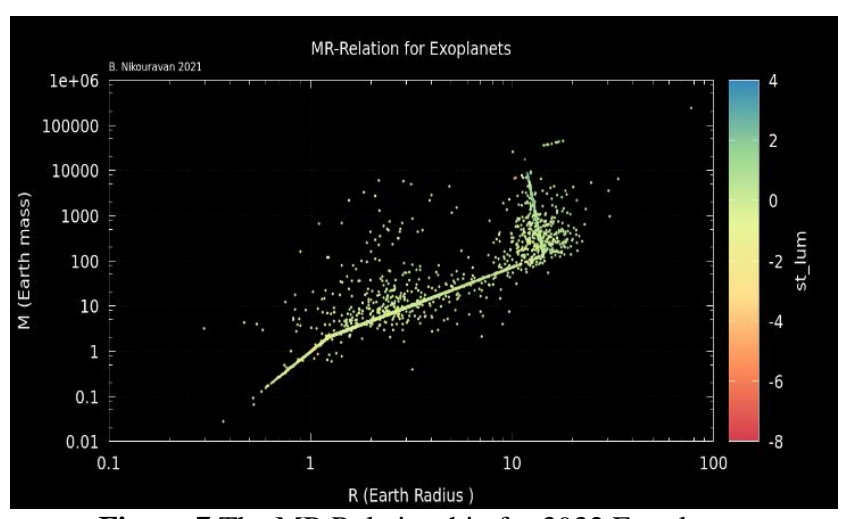

Figure 7 The MR Relationship for 3932 Exoplanets

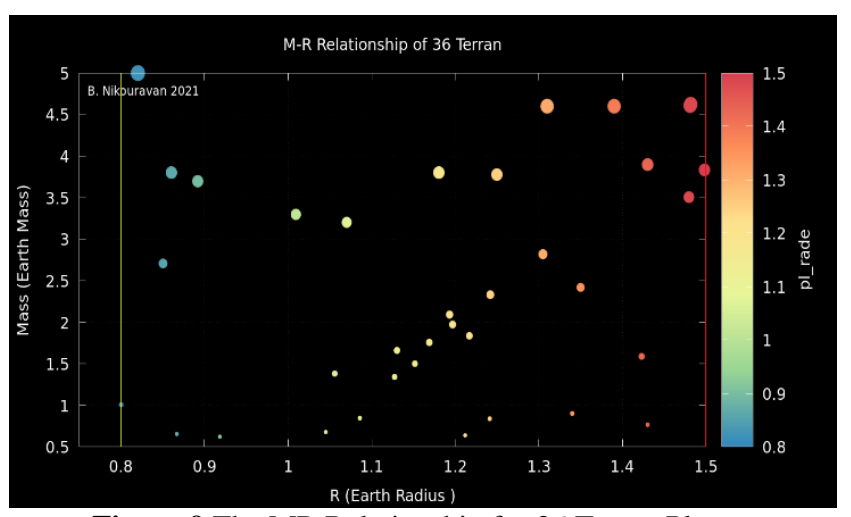

Figure 9 The MR Relationship for 36 Terran Planets

\section{EXPANDING MR-RANGE}

The aim here is to find a broader, possible catalog; expanding the mass-radius relationship of exoplanets in a wider range of masses between 0.1 to 100 Earth-mass and radius from 0.4 to 15 Earth radius. This area is not only necessarily a region for potentially habitable exoplanets, but also can covers all exoplanets with different mass and radius.

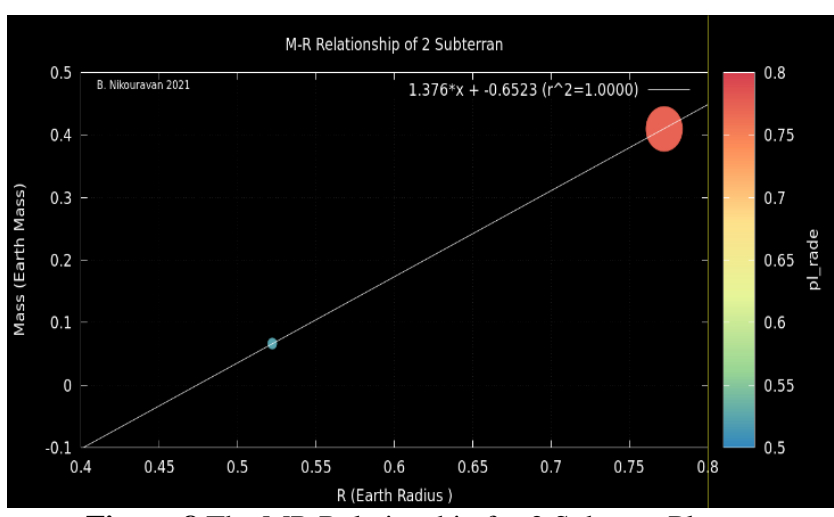

Figure 8 The MR Relationship for 2 Subtrran Planets

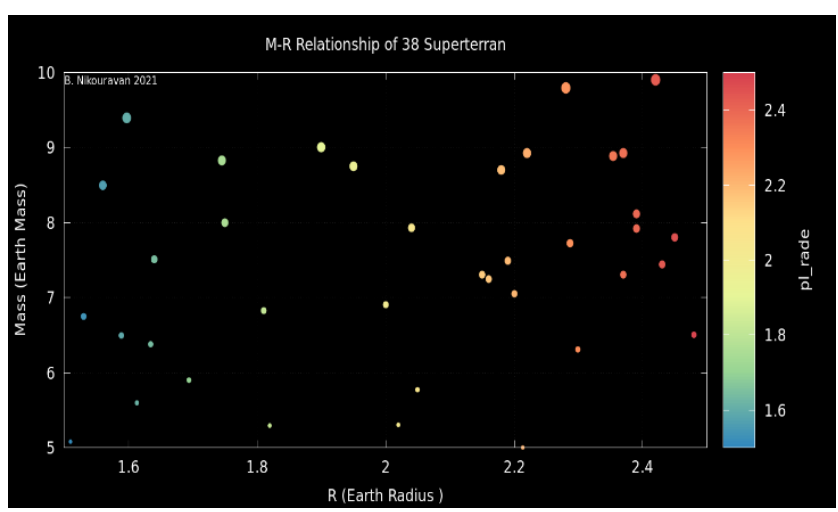

Figure 10 The MR Relationship for 38 Superterran Planets

The results were obtained empirically by the best fitting point of regression values of the M-R relationship in 7 groups with a strong positive correlation between mass and radius for each group. (see Figures 10 to 17). Initially, we have found 2531 confirmed exoplanets out of 4383 , with masses between 0.1 to $100 M_{\oplus}$ and radius between 0.4 to $15 R_{\oplus}$ (by End of April 2021) with a very strong correlation. (Table 7).

Table 7 A cataloging of Exoplanets in mass and radius between 0.1 to $100 \mathrm{M}_{\oplus}$ and radius between 0.4 to $15 \mathrm{R}_{\oplus}$

\begin{tabular}{ccccc}
\hline No & $\mathrm{M}\left(M_{\oplus}\right)$ & $\mathrm{R}\left(R_{\oplus}\right)$ & $R^{2}$ & No of Planets \\
\hline 1 & $0.1-0.2$ & $0.4-0.8$ & 0.9984 & 5 \\
2 & $0.21-1$ & $0.8-1$ & 0.9929 & 94 \\
3 & $1-2.8$ & $1-1.4$ & 0.9847 & 409 \\
4 & $2.8-5.5$ & $1.4-2.2$ & 0.9507 & 743 \\
5 & $5.5-10$ & $2.2-3.2$ & 0.9373 & 786 \\
6 & $10-17.5$ & $3.2-4.5$ & 0.9511 & 255 \\
7 & $20-100$ & $4.5-15$ & 0.9129 & 239 \\
\hline
\end{tabular}




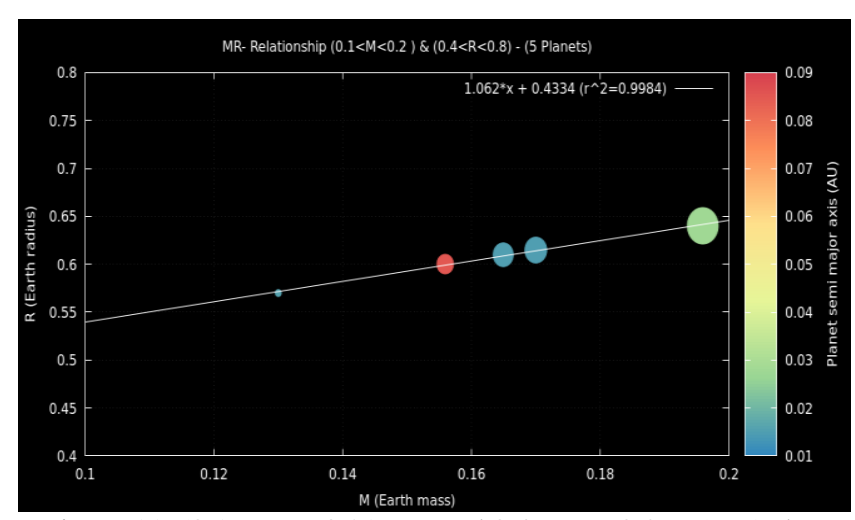

Figure $11\left(0.1<\mathrm{M}<0.21 M_{\oplus}\right.$ and $\left.0.4<\mathrm{R}<0.8 R_{\oplus}\right)-5$ Planets

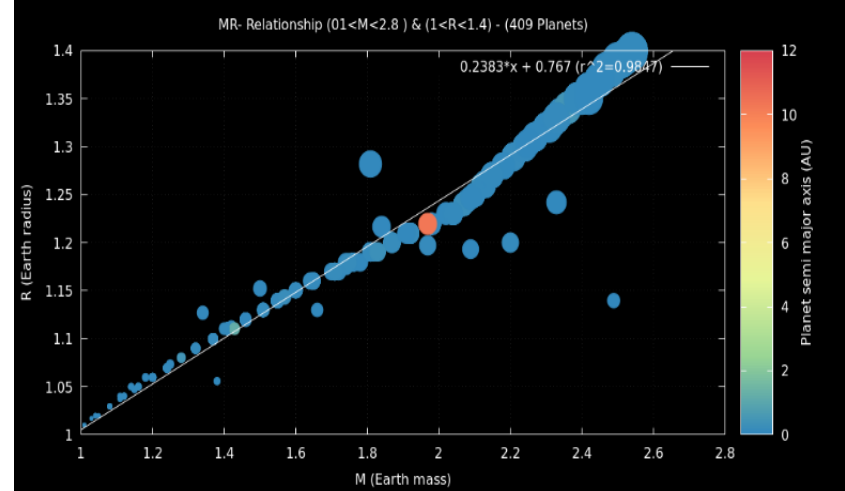

Figure $13\left(0.1<\mathrm{M}<2.8 M_{\oplus}\right.$ and $\left.1<\mathrm{R}<1.4 R_{\oplus}\right)-409$ planets MR-Relationship $(5.5<M<10) \&(2.2<R<3.2)-(786$ Planets $)$

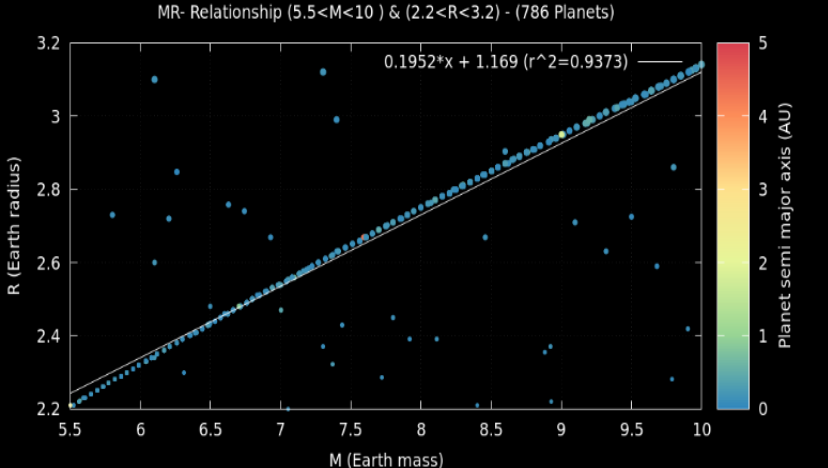

Figure $15\left(5.5<\mathrm{M}<10 \mathrm{M}_{\oplus}\right.$ and $\left.2.2<\mathrm{R}<3.2 \mathrm{R}_{\oplus}\right)-786$ planets

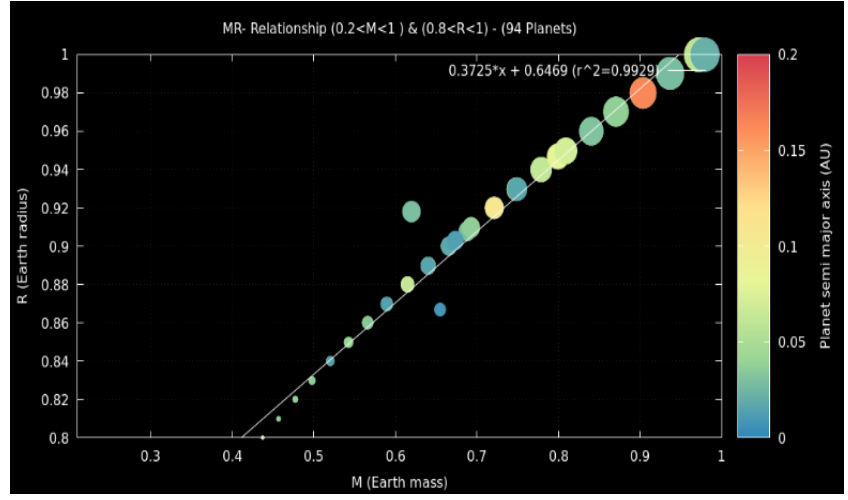

Figure $12\left(0.2<\mathrm{M}<1 M_{\oplus}\right.$ and $\left.0.8<\mathrm{R}<1 R_{\oplus}\right)-94$ planets MR- Relationship $(2.8<M<5.5) \&(1.4<R<2.2)-(743$ Planets $)$

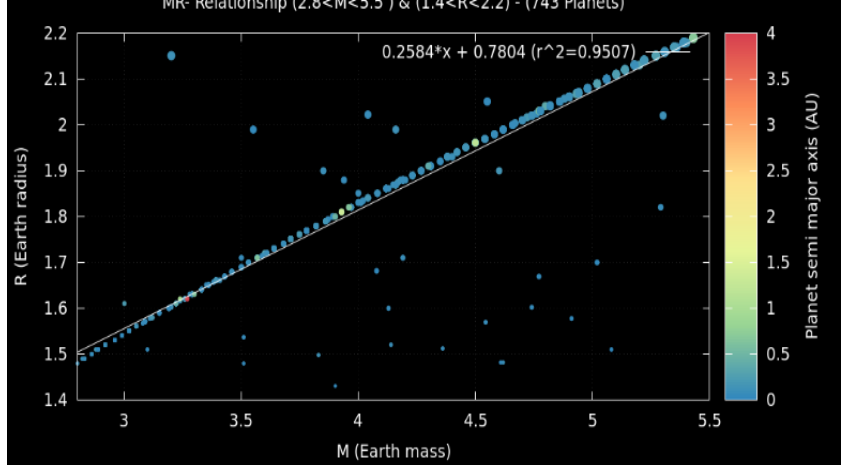

Figure $14\left(2.8<\mathrm{M}<5.5 M_{\oplus}\right.$ and $\left.1.4<\mathrm{R}<2.2 R_{\oplus}\right)-743$ planets

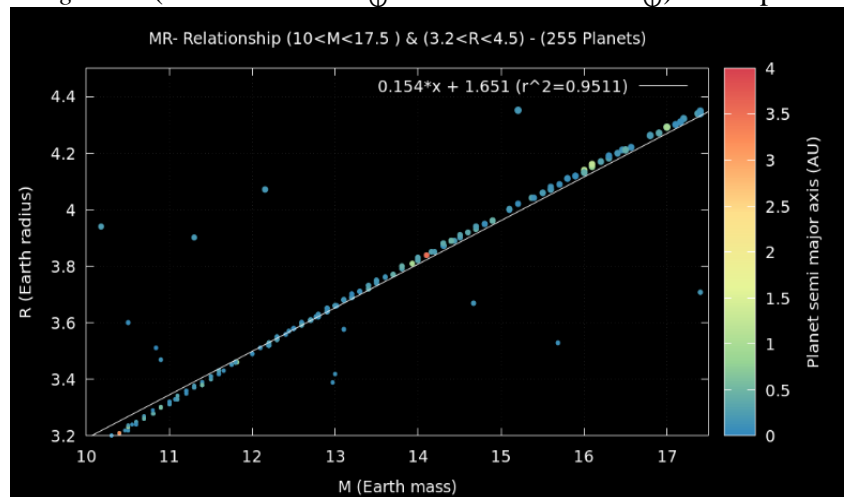

Figure $16\left(10<\mathrm{M}<17.5 \mathrm{M}_{\oplus}\right.$ and $\left.3.2<\mathrm{R}<4.5 \mathrm{R}_{\oplus}\right)-255$ planets

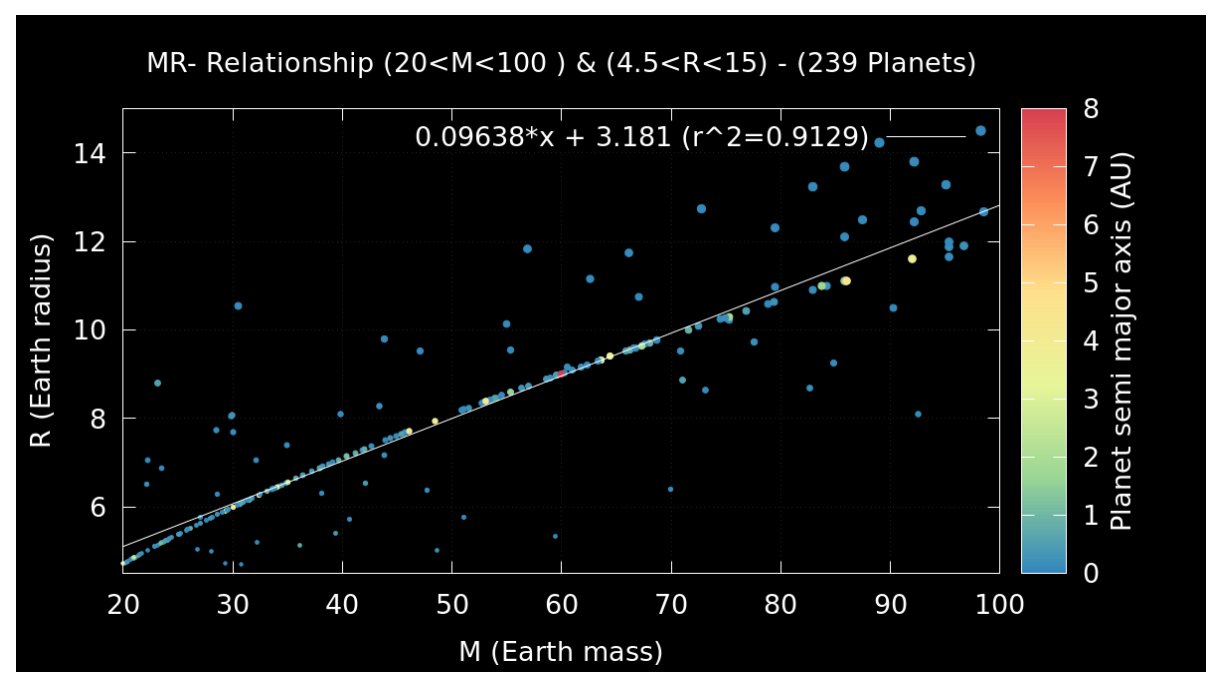

Figure $17\left(20<\mathrm{M}<100 \mathrm{M}_{\oplus}\right.$ and $\left.4.5<\mathrm{R}<15 \mathrm{R}_{\oplus}\right)-239$ planets 


\section{DISCUSION AND CONCLUSIONS}

In this work, we have calculated the following essential physical properties of confirmed exoplanets, including potentially habitable exoplanets.

The Semi-major axis for 35 potentially habitable confirmed planets by applying Kepler's Third Law. In this calculation, the minimum and maximum mass and radius of planets are between 0.5 and 5 Earth-mass and 0.8 and 1.5 Earth radius respectively. The calculation of the semi-major axis shows a good correlation with the original data collected from NASA Exoplanets Archive (Table 1).

Figure 1 and 2 show a good connection between observed and calculated values among the semi-major axis and orbital period with $R^{2}=0.8101$ and $R^{2}=0.9314$, respectively. Consequently, the probability density function of the calculated semi-major axis Histogram for 35 planets, shown in Fig 4.

Using the previous calculation for the semi-major axis of habitable planets, the known mass of the star, the orbital period of a planet around the center of mass of its star, and combining Equation 3 and radial velocity amplitude, the mass of the exoplanets has been estimated. Determined masses are around the mass of solar, Jupiter and Earth mass. The results of this calculation (Table 2) shown a strong linear relationship between the theoretical calculation and observation.

The next important physical quantity of brightness is the luminosity of the star. The luminosity of a star can be calculated in two different ways. One is with using the bolometric magnitude of the star, and /or using the radius and temperature of the star which have been selected. The estimated values are shown in Table 3 with a very strong correlation.

Habitable Zone (HZ) is the range of orbits around a star in three regions, inner, center, and outer. Using the previous calculation for the luminosity, the inner, center, and outer region of 3522 planets out of 3945 approved exoplanets, simply calculated. Table 4 show only for a 40 exoplanet. In this region, a planetary surface can support liquid water given sufficient atmospheric pressure. The inner and outer boundaries are defined from Venus to Mars models, respectively. For a Sun-like star (G2), these are given at 0.75

\section{ACKNOWLEDGEMENT}

I want to especially thank to Prof J. J. Rawal for preliminary revision of the manuscript and his suggestion and corrections. I thank the Planetary Habitability Laboratory (PHL) which is a research and educational virtual laboratory, University of and 1.77AU. To estimate $\mathrm{HZ}$, we neglect the stellar effective temperature and define the center of $\mathrm{HZ}$ as $1 \mathrm{AU}$ for Earth around the Sun, and also scale with stellar luminosity.

Here we have calculated the radial velocity amplitude (RVA) of each exoplanet with its estimated mass in the previous calculation. The predicted radial velocity amplitude depends on the planet orbital period, planet mass, and stellar mass, and the semi-major axis of the planet. Table 4 shows accurately calculated values for $K$, in $\mathrm{m} / \mathrm{s}$.

The planetary equilibrium temperature (PET) is a theoretical temperature that a planet would be a black body being heated only by its parent star and it can be determined by the size, temperature, and distance to its parent star, but not on its size. Here, to calculate PET, we need the radius of the star, the distance of the star from the planet, the amount of Albedo, and finally, the temperature of the sun. Albedo is the fraction of energy per unit of time that is reflected by the planet and it is a value between 0 and 1 and with no dimension in the unit.

Planet density is the most important physical property of planets. Typically, planet density tells us what a planet is made of and how much material it has in space. This property simply can be calculated with the mass and radius of the planet. Normally planets are in a different wide range of sizes and masses but planets made of the same material will have the same density regardless of their size and mass.

According to the up-to-date values, we have found a total number of 76 planets between $\left(0.1 \leq \mathrm{M}_{\mathrm{p}} \leq 10\right) \mathrm{M}_{\oplus}$ and $(0.4 \leq \mathrm{R} \leq 2.5) \mathrm{R}_{\oplus}$, precisely with 2 planets in the first region, 36 planets in the second region, and 38 planets in the third region.

Certainly, classification of exoplanets can help to find more information about the planet and its properties. Here cataloging planets in mass and radius have been done for a total number of 2531 exoplanets in 7 groups with a strong correlation for each group respectively (Table 7). All data collected from the NASA Exoplanet Archive database, (NASA Exoplanet Archive, 2021) by the end of April 2021.

Figures 11, to 17 show a very interesting relationship between mass and radius of planets in the different mass and radius with the highest linear correlations.

Puerto Rico at Arecibo, dedicated to studies of the habitability of Earth, the Solar System, and exoplanets. All data are made available using services at the NASA Exoplanet Archive, which is operated by the California Institute of Technology, under contract with the National Aeronautics and Space Administration under the Exoplanet Exploration Program. 


\section{REFERENCES}

Akeson, R., Chen, X., Ciardi, D., Crane, M., Good, J., Harbut, M., . . Leifer, S. J. P. o. t. A. S. o. t. P. (2013). The NASA exoplanet archive: data and tools for exoplanet research. 125(930), 989.

Alibert, Y. J. A., \& Astrophysics. (2014). On the radius of habitable planets. 561, A41.

Baraffe, I., Chabrier, G., \& Barman, T. J. R. o. P. i. P. (2009). The physical properties of extra-solar planets. 73(1), 016901.

Bashi, D., Helled, R., Zucker, S., Mordasini, C. J. A., \& Astrophysics. (2017). Two empirical regimes of the planetary mass-radius relation. 604, A83.

Kasting, J. F., Whitmire, D. P., \& Reynolds, R. T. J. I. (1993). Habitable zones around main sequence stars. 101(1), 108-128.

Malhotra, R. J. T. A. J. (2015). The mass distribution function of planets. $808(1), 71$.

Müller, S., Ben-Yami, M., \& Helled, R. J. a. p. a. (2020). Theoretical vs. observational uncertainties: composition of giant exoplanets.
NASA Exoplanet Archive. (2021). Retrieved from: https://exoplanetarchive.ipac.caltech.edu/

Perryman, M. A. J. R. o. P. i. P. (2000). Extra-solar planets. 63(8), 1209.

Planetary Habitability Laboratory. (2021). The Habitable Exoplanets Catalog. http://phl.upr.edu/

Raymond, S. N., Quinn, T., \& Lunine, J. I. J. A. (2007). High-resolution simulations of the final assembly of Earthlike planets. 2 . Water delivery and planetary habitability. 7(1), 66-84.

Seager, S., Kuchner, M., Hier-Majumder, C., \& Militzer, B. J. T. A. J. (2007). Mass-radius relationships for solid exoplanets. 669(2), 1279.

Stull, R. B., \& Ahrens, C. D. (2015). Meteorology for Scientists and Engineers, 3rd Edition: Brooks/Cole, Page 794.

Weiss, L. M., \& Marcy, G. W. J. T. A. J. L. (2014). The mass-radius relation for 65 exoplanets smaller than 4 Earth radii. 783(1), L6. 